Ho:pital Arriurin.

Cátidta da Pestiatria.

Prof. E. Cienfuegos.

Savicio de Radiologia.

\title{
CONTROL PULMONAR EN LA OSTEOARTRITIS TUBERCULOSA
}

\section{Por al Dr. EFRAIN VOLOSKY}

Por insinuación de1 Dr. Guillermo Valenzuela y con su colaboración, hemes llevado a efecto este trabajo, cuyo objeto principal es ubicar la osteoartritis dentro de la enfermedad tuberculosa.

Si consideramos que la osteoartritis tuberculosa no debe clasificarse como enfermedad regional del sistema loconotor. tampocc caeremos en la exageración opuesta de descuidar d tratamiento ortopédicc y actuar sólo con medidas generales.

En relación con su localización, la osteoartritis entra en $e^{1}$ grupo de las tuberculosis extrapulmonares, del cual forma parte principal: le siguen en orden de frecuencia las adentis tuberculosas y la tuberculosis urcgenital.

De acuerdo con su evolución arrastrada can tendencia a ta localización, se considera a la osteoartritis como una tuberculosis de tipo terciario.

En este estudio quisimos averighar la frecuencia de las lesiones pulmonares en ia tuberculosis osteoarticular. La in. verea del problema, o sea. saber la proporción con que la tuberculcsis fulmonar se complica de osteoartritis, es algo más largo y dificil de averiguar y al respecto sólc podemos citar aigunas cifras. En Chile, revisadas 4.000 autopsias hechas en el Hospital San José. en un $4 \frac{1}{\text { k }}$ de los casos había complicaciones de osteitis u osteoartritis. Hanum y Meker, estudiando 1.864 tuberculcsos, comprobaton que en el $2 \%$ de los casos presentaron durante su evolución lesiones óseas. 
Condíciones del trabajo. - Se reunieron 100 casos de ostecartritis y osteitis tuberculcsa. debidamente comprobadas. sin tomar en cuenta su localización. La edad de los niños fluctuó entre 1 y 11 años.

Cada enfermo debía tener una radiografía pulmonar por la menos y que hubiera sido tomada antes del año de iniciada la afecrión ósea: sin embargo. en 20 casos se hizo después del año. La mitad de los enfermos se siguió perscnalmente. el resto de los casos se obtuvo de los archivos del Hcspital.

La gran mayoría de los enfermos, 94. ennsultó por su afección articular u ósea en la Policínica de Cirugía y sólo 6 fueron enviados en consulta desde la Policlínica de tubercilosis donde estaban inscritos per slguna lesión pulmonar.

Edad de los enfermos.

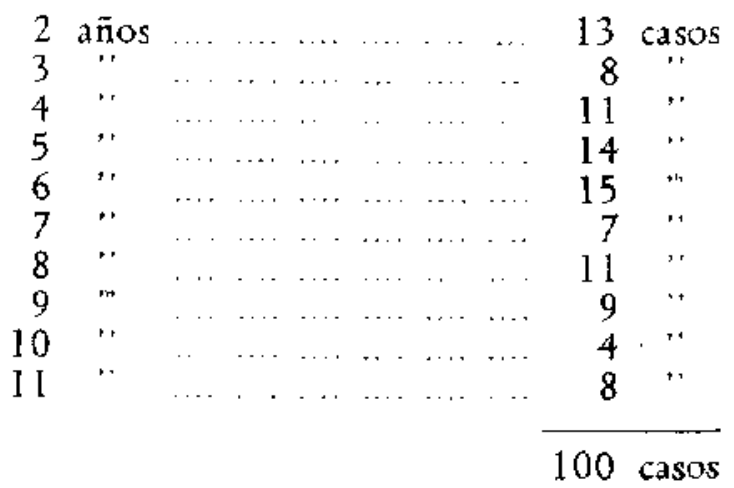

Tipo de lesión ósea.

Mal de Pott

Coxalgia

21 cas os

Ostecartritis rodilla

Osteoart :itis ribiotarsiana

Espina ventosa

Trocanteritis

Osteoartritis codo

33

34

5 "

4 .

2 "

100 casos

Lesiones pulmcnares encontradzs: Las radiografías pulmonares pueden clasificarse en tres grupcs. cuya frecuencia va a continuación: 
Radiografias negativas 19 casos

Radiografias con lesiones residuales

Radiografías con lesiones activas

El detalle del segundo y tercer grupa es el siguiente:

L.csiones residuales: complejos $1 .^{\circ}$ calcificados, adenopatias calcificadas y nódulos calcificados

12 casos campos de induración pulmo. nar o hiliares pleuritis cicatricial

5 " 3 "

20 casos

Lesiones activas: adenopatías complejos primario: 25 casos infiltrados 2 diseminaciones hem a tóge nas puras pleuritis y derrames $\begin{array}{r}6 " \\ . \\ 3 " \\ \hline 61 \text { casos }\end{array}$

diseminaciones he matógenas asociadas a otras lesiones ya descritas

\section{1 casos}

Como era de preverlo, por la edad de los niños, sóito se encontró formas de primoinfección o sus complicaciones inmediatas, como ser diseminaciones y pleuritis.

En 11 de los casos las lesiones pueden calificarse como extonsas, de ellos 8 corresponden a diseminaciones hematógenas de mediana o gran intensidad.

De los 21 niños con complejo primario, 14 de ellos presentan francos signos de regresión en forma de calcificación o tendencia a la induración y sólo 7 estaban en plena evolución. De las adenoparías, en 8 casos se trata de lesiones muy pequeñas, semiinduradas o en vías de calcificación y el resto si no presenta caracteres excesivamente benignos tampoco son de aspecto alarmante.

Revisando las carátulas se comprueba otro hecho de interés: mientras menor es el niño, más ftecuentes y más graves son las lesioncs pulmonares encontradas; en cambio, en los niños mayores éstas son más benignas. 
Relación entre la edad' y la frecuencia de lesicones pulmonares.

\begin{tabular}{llccc} 
& $1.5 \cdot 4$ años & 5.7 años & $8-11$ años \\
Con lesión pulmonar activa & 26 casos & 21 casos & 14 casos \\
Sin lesión pulmonar & $2 .$. & 6 & 15 & 18 \\
\hline 32 & 36 & $32=$
\end{tabular}

100 casos.

Llama la atención, también. que en los niños con más de una lccalización ósea, son más frecuentes y extensas las manifestaciones pulmenares.

Evolución y pronóstico de la lesión pulmonar. - En 22 enfermos con manifestaciones activas pulmonares hemos podido seguir su evolución, siendo favcrable en la gran mayoria. 20 casos. y desfavorable en 2. Estc contrasta con el curso más prolongado y expuesto a complicacicnes. como ser supuración, fistulización. etc., que experimenta la lesión ósea. Podríd resumirse estc diciendo que la parte pulmonar evoluciona como tuberculosis primaria y la ósea como terciaria.

Esta misma tendencia favorable de la lesión pulmonar se aprecia en el gran número de casos en que ya durante el primet examen bay signos de curación, como ser la tendencia a la cáleificación.

Sin embargo, desde el punto de vista pronóstico, sería un grave error afirmar la benignidad de las lesiones encontradas. La pequeñez de un ganglio o su aparente calcificación no es inconveniente ninguno para que en cualquier momento provoque una diseminación. El hecho que varios de nuestros enfermos con primoinfección extensa y diseminaciones graves bayan aparentemente regresado, si bien tos permiten hacer un pronóstico inmediato favorable, nada nos dice del futuro. basta sclamente recordar a este tespecto el aforismo de Rafdecker, que dice que "a mayor gravedad de la primoinfección. mayores posibilidades de reinfección".

Epcra de aparición de la osteloartritis. - Aunque la ostecartritis puede aparecer en cualquier periodo de la tuberculosis, tiene predilección por ciertas fases. Según Wallgreem. e1 peligro mayor de la osteoartritis es su aparición dentro de los dos primetos años de la primoinfección: pasados los tres añcs, las posibilidades son muy escasas. De acuérdo con este principio de Wallgreem, la mayoría de nuestros enfermos presentaba lesiones pulmonares frescas, Io que estaba indican- 


\section{Evolución de la lesión pulmonar.}

DEST:AVORABLE

1. C. 7 años i:r $1 X-41$ Complejo $l$ "alcificado.

In X -41 Sè agrega pleuritis basal der.

1. F. 7 sños En X -39 Adenopatia hiliar.

I:ת IX -40 Diseminación biłatzrá.

IAVORABLE

E. M. o años lin III $\$$ () Complejo l." der. en svaluión.

i.o Vl - +1 Calcificación base der, adenopatia en calvificusción.

‥ A. 2 años $\Gamma n X I+2$ Infiltrato l." rercio superior dzr

L.' IV t +3 Pleuricis mediastinisa der.

C. F. $\quad$ años $[$ กn X] 42 Pleuritis marginal inf. der. probable adenopalia.

lia VI -42 Tórax normal.

B. A. + años [n $1 V-4]$ Complejo $1 \%$ iz en enolución. Ta leariulosis disemirada distrera.

En VII -4? Adenopatia hiliar iza. indurada.

A. $1 . \quad 6$ añs $\quad$ En $X$.38 Tuberculosis dieminada bilar, y adenopartia biliaz izq.

Lir $1 X-40$ Disminaciones zusi completamente calcific, y ganglios hiliares y paraltay calcif.

F. $1 . \quad$ añ IX -4 Adenopatía hiliar der

Eir Il. -43 Hilio Jer. indurado.

A. F: 7 ingos IV -42 Infiltrado parahiliar inf. irq

Li; Vlil-43 Proceso infillativo izq. en curación.

I. C. 3.5 2ños t. VI -42 Infiltado direcho en esbsorción.

En IX +2 Lesiones inactivas in vias de calcific.

D. M. 6 ati En IX -38 Complejo l." dir. en evoluión.

F.n XI -30 Adenopatia es vias de colcificación.

En V -43 Calcificación paratraqueal der.

J. S. 2 años L̈: Il 40 Adenopatia hilias izk.

En Il -4; Torax norma!

R. Y. 4 añs En II -4] Tuberculo: is mixta biaterat.

Iin $X I-41$ Tubercul. productiva indarativa bilat.

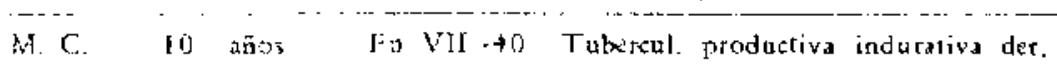

Fo IV .4I Sombras mos inducadas. 


\begin{tabular}{|c|c|c|c|}
\hline R. $T$. & + tios & $\begin{array}{l}\text { En VI }-40 \\
\text { En III }-41\end{array}$ & $\begin{array}{l}\text { Cemplejs } 1 . " \text { der. en evoiución. } \\
\text { Primoinfección cieatrizada. }\end{array}$ \\
\hline J. C. & 5 años & $\begin{array}{l}\mathrm{E} \pi \mathrm{VIII}-40 \\
\mathrm{l}-\mathrm{X} \text { XII }-42\end{array}$ & $\begin{array}{l}\text { Compleju l." izq. en vias de catit. } \\
\text { y III-4 Complejo l." izq calcif. }\end{array}$ \\
\hline E. A. & 3 มี่อก & $\begin{array}{l}\operatorname{In} \text { XII }-40 \\
\text { En } 1 I \quad+1 ! \\
\text { En VII }-43\end{array}$ & $\begin{array}{l}\text { Derrame ficural dar. } \\
\text { Paquiplauritis. } \\
\text { Tórax nomal. }\end{array}$ \\
\hline S. P. & מחה & $\begin{array}{lll}\text { fin } & \text { III } & -+1 \\
\text { Iir } & {[X} & -43\end{array}$ & $\begin{array}{l}\text { Adenopatia biliar fereiha. } \\
\text { Hilios dinsos. }\end{array}$ \\
\hline H. Z. & \& años & $\begin{array}{lll}\text { Er } & 1 & + \\
\text { Er } & V & +11\end{array}$ & $\begin{array}{l}\text { Dirame pletaral dericho. } \\
\text { Pacuiphleuritis costediafagmatica der. }\end{array}$ \\
\hline A. $M$. & 2 años & $\begin{array}{l}\operatorname{lin} 11 \mathrm{i}-4 j \\
\text { En VIIT-43 }\end{array}$ & $\begin{array}{l}\text { Adenopazia hiliar izq. } \\
\text { Tórax normal. }\end{array}$ \\
\hline L. $A$. & 6 iño3 & $\begin{array}{l}\text { En IV } \\
\text { En VI } \\
\end{array}$ & $\begin{array}{l}\text { Complejo I." derecho en regnaión, } \\
\text { Adenopalia faratraqueal der. calcif. }\end{array}$ \\
\hline N. A. & 2.5 años & $\begin{array}{l}\text { in XII } 43 \\
\text { En XI } 43\end{array}$ & $\begin{array}{l}\text { Primcinfacción der pabablementi ex- } \\
\text { cavada. } \\
\text { Aderopatia hiliar y faractaqual der. }\end{array}$ \\
\hline
\end{tabular}

do la cercanía dé contago en otroi casos (12). el examan pulmcnar nos reveló lesiones calcificadas, lo que sólo nos permite afirmar que han transcurrido más de dos años desde la primcinfección, tiempo que se calcula como ef mínimo que tarda en calcificar ésta. Tenemos dos observaciones en que la astecartritis apareció a los 5 años de comprobada la primoinfección, a pesar de haber curado bien desde el punto de vista radiclógico.

La edad es un factor de importancia en cuanto se reficie a la época de aparición de la cstecartritis: mientras menor es e! niño, más precozmente aparecen las localizaciones articalares y más tendencia tienen a la multiplicidad. Estas metástasis óseas estatían en relación con diseminaciones precoces del período primario.

Necesidad del estudic radiogtáfico del pulmón. Aunque es un becho por demás repelido, conviene insistir en ia necesidad del estudio mixto radioscópico radiográfico del pulmón. El conformarse ccn él control radioscópico, puede canducir a error. la radiografía es su complemento indispensable, fermitiende precisat ciertas lesiones, como set diseminaciones o infiltrados tenues que escapan a aquél. En el cass de los pacientes osteoarticulares, aumentan los inconve- 
nientes de la radioscopía, dado que estando en general enyesados, ra pueden verse con la calma y en las posiciones necesarias.

En nuest as observaciones, en 10 casos habia diferencias notorias entre ambos métodos de examen.

\begin{tabular}{|c|c|c|c|c|c|c|}
\hline Nombte & & Edad & Radioscopia & Radiogr & rafía & \\
\hline গ. A. & 7 & años & Negativa & Adenopatiz biliar izq. & • & \\
\hline B. A. & 4 & มี่อร & Negativa & $\begin{array}{l}\text { Complejo } 1^{9} \text { izq. } \\
\text { discreta. }\end{array}$ & Tiberculosis & diseminad \\
\hline
\end{tabular}

E. M. 7 ẵos Negativa Adenopatia hiliar izq.

E. C. 4.5 años Negativa Adenopatia hiliar y paratraqueal der. infiltrado der. en reabsosción.

C. L. 4 años Negativa pleuritis mediastinica iza. superior.

M. F. 7 años Negativa Infiltrado parahiliar der en reabsorción, dístminación hematogena discrata.

S. N. 4 años Negativa Adenopatía hiliar iza. superior.

O.C. 1l años Negativa Pleuritis adbesiva cicatricial ambos biatos costodiafragmáticos. Induración vírtice der.

P. P. 4 años Negativa Adenopatia hiliar bilateral.

M. D. 8 años Adenopatía Algunos nódulos calcíficados en el tercio tnehiliar iza. dio derecho.

Comparación con el adulto y el adolescente. - Si bien es cierto que en el adulta son tan frecuentes como en el niño las lesiones pulmonares en cásos de osteoartritis tuberculosas. la importancia y el pronóstico de aquéllas difiere en ambos casos. Es notorio que en el adulto y el adolescente es más grave la parte pulmonar; en un 50 a $55 \%$ de los que padecen tuberculosis ósea se encuentra comprometido el pulmón: en el $30 \%$ del total de los casos, la tuberculosis pulmorar se califica de extensa. La evolución. por otra parte, es mucho más desfavorable que en el niño. Alonso Vial, estudiando 105 adultos y adolescentes que tenían osteoartritis asosiada con tuberculosis pulmonar, comprueba que en 48 casos la lesión pulmonar progresó en 26 mejoró y 18 fallecieron.

Es notable el hecho de que aún las formas de primoinfrcción evolucionan desfavarablemente. en el adulto. El 
mismo autor. estudiando 12 enfermos osteoarticulares con tuberculosis pulmonar de primoinfección, vió que ésta se agravó en 6 casos, aparte de otros dos que fállecieron.

Importancia del control pulmonat en la tuberculosis osteoarticular. - En toda afección ósea de naturaleza oscura, el hallazgo de lesiones pulmonares, sean residuales o activas, éstas con mayor razón, servirán para aclarar el origen tuberculoso de aquélla. Pasa a veces que en un comienzo ia osteoartritis no da signos radiológicos y se descuida su tratamiento, si en estos casos se estudiasen los demás signos de tuberculosis: Mantoux. sedimentación, radiografía pulmonar. etc., ya habría más base para el diagnóstico y no se perdería un tiempo precioso.

La importancia pronóstica del control pulmonar está clara. en un $10 \%$ de las osteoartritis se encuentran formas extensas de cuberculosis pulmonar. que por sí solas son un peligro para la vida del enfermo y que favorecen la aparición sucesiva de metástasis óseas. La muerte se produce generalmente por diseminaciones masivas, cuyo diagnóstico precoz. como también su punto de partida, en general lo gan. glios paratraqueales, lo da la radiografía pulmonar.

E1 valot principal del control pulmonar es el de fijar el criterio terapéutico, determinando cuál es el órgano principalmente afectado, si es el pulmón o la articulación. sin olvidar en ringún caso las reglas generales del tratamiento an. rituberculoso.

\section{Conclusiones.}

1. Es indispensable el estudio radiológico pulmonar en todo enfermo. sospechose o confirmado, de osteoartritis tabejculosa.

2." En el $61 \%$ de los enfermos con tuberculosis ósea o articular, encontramos lesiones pulmcnares en actividad. del tipo de la primeinfección o sus complicaciones inmediatas.

3. En el $11 \%$ de los casos, las lesiones pulmonares erin extensas.

4. Mientras menor es el niño, más frecuente y más grave es el comproniso pulmonar.

5. El pronóstico inmediato de la tuberculosis pulmonar, en general, fué favorable. como corresponde a las formas de primoinfección. 